\title{
Licochalcone $\mathbf{H}$ induces the apoptosis of human oral squamous cell carcinoma cells via regulation of matrin 3
}

\author{
SU-HYUN NHO ${ }^{1 *}$, GOO YOON ${ }^{2 *}$, JI-HYE SEO ${ }^{1}, \mathrm{HA}^{*}$ A OH ${ }^{2}$, SEUNG-SIK CHO $^{2}$, HANGUN KIM $^{3}$, \\ HYUN WOO $\mathrm{CHOI}^{4}$, JUNG-HYUN SHIM ${ }^{2,5}$ and JUNG-IL CHAE ${ }^{1}$ \\ ${ }^{1}$ Department of Dental Pharmacology, School of Dentistry and Institute of Oral Bioscience, BK21 Plus, \\ Chonbuk National University, Jeonju, Jeollabuk 54896; ${ }^{2}$ Department of Pharmacy, College of Pharmacy \\ and Natural Medicine Research Institute, Mokpo National University, Muan, Jeonnam 58554; \\ ${ }^{3}$ College of Pharmacy and Research Institute of Life and Pharmaceutical Sciences, Sunchon National University, \\ Sunchon, Jeonnam 57922; ${ }^{4}$ Department of Animal Science, Chonbuk National University, Jeonju, Jeollabuk 54896, \\ Republic of Korea; ${ }^{5}$ The China-US (Henan) Hormel Cancer Institute, Zhengzhou, Henan 450008, P.R. China
}

Received January 10, 2018; Accepted September 4, 2018

DOI: 10.3892/or.2018.6784

\begin{abstract}
Licochalcone $\mathrm{H}(\mathrm{LCH})$ is a chemical compound that is a positional isomer of licochalcone C (LCC), a chalconoid isolated from the root of Glycyrrhiza inflata, which has various pharmacological properties including anti-inflammatory, antioxidant, antitumor, and anticancer effects. However, the efficacy of LCH on cancer cells has not been investigated. The present study examined the effects of LCH on cell proliferation, induction of apoptosis, and the regulation of matrin 3 (Matr3) protein in oral squamous cell carcinoma (OSCC) cells by Annexin V/propidium iodide (PI) staining and western blot analysis. $\mathrm{LCH}$ reduced cell viability and colony forming ability, and induced cell cycle arrest and
\end{abstract}

Correspondence to: Professor Jung-Hyun Shim, Department of Pharmacy, College of Pharmacy and Natural Medicine Research Institute, Mokpo National University, 1666 Youngsan-ro, Muan, Jeonnam 58554, Republic of Korea

E-mail: s1004jh@gmail.com

Professor Jung-IL Chae, Department of Dental Pharmacology, School of Dentistry and Institute of Oral Bioscience, BK21 Plus, Chonbuk National University, 567 Baekje-daero, Jeonju, Jeollabuk 54896, Republic of Korea

E-mail: jichae@jbnu.ac.kr

${ }^{*}$ Contributed equally

Abbreviations: OSCC, oral squamous cell carcinoma; $\mathrm{LCH}$, licochalcone H; DMEM, Dulbecco's modified Eagle's medium; FBS, fetal bovine serum; DAPI, 4'-6-diamidino-2-phenylindole; $\mathrm{P} / \mathrm{S}$, penicillin and streptomycin; PBS, phosphate-buffered saline; PI, propidium iodide; siRNA, small interfering RNA; siMatrin3, matrin 3-specific targeting siRNA

Key words: licochalcone $\mathrm{H}$, matrin 3, oral squamous cell carcinoma, apoptosis apoptosis in HSC2 and HSC3 cells through the suppression of Matr3. It was also found that LCH directly bound to Matr3 in a Sepharose 4B pull-down assay. Consequently, the results of the present study suggest that $\mathrm{LCH}$ may be used as an anticancer drug in combination with conventional chemotherapy for the treatment of OSCC, and that Matr3 may be a potential effective therapeutic target.

\section{Introduction}

According to the World Health Organization, cancer is the second leading cause of mortality worldwide, with $8,800,000$ individuals succumbing to mortality in 2015. Statistically, this means that one in six individuals succumbed to cancerassociated mortality worldwide in 2015. The incidence of cancer is increasing due to changes in life patterns, economic development, urbanization, population growth, and aging (1).

Oral cavity cancer and pharyngeal cancer represent the sixth most common types of cancer in the world, and occur more frequently in men than in women (2). According to data from the Korea Central Cancer Registry, a high number of individuals aged between 50 and 70 years have oral cancer; the incidence of oral cancer has also been increasing in the younger generation in recent years. Oral squamous cell carcinoma (OSCC) is a malignant tumor, which accounts for $>90 \%$ of all cases of oral cancer (3). In terms of the distribution of oral cancer sites, tongue cancer accounts for the highest proportion, $\sim 45 \%$ (4). In general, the 5-year survival rate of individuals with tongue, oral cavity, and tonsillar cancer is between 50 and 55\%, which is comparatively lower than that of other types of cancer (5). Since 2016, 3,236 cases of oral cancer have been reported in Korea, 1,059 cases of which resulted in patient mortality (6). Various types of oral cancer treatment, including surgery, radiation therapy and chemotherapy, are typically used to treat patients. However, the majority of these methods are accompanied by side-effects and recurrences (7).

Natural products have been used in medicine since early human history (8). Generally, they are known to have low 
toxicity, are readily accessible, and have a fairly wide range of application methods (9). Although the development of natural drugs has been continuously expanding, few cases have been reported in terms of their development as anticancer drugs (10). Therefore, natural drugs require consideration for cancer therapy (11). For example, the medicinal herb licorice has been commonly used in China for a number of centuries and has various pharmacological effects, including anticancer, anti-inflammatory, anti-ulcer, and antibacterial effects (12). To date, seven retrochalcones, which are structurally unique and distinguished from ordinary chalcones at the C-2' and C-6' positions, comprising licochalcone (LC) A-E, LCG and echinatin, have been isolated and characterized from the roots of Glycyrrhiza inflata, with the exception of the artificial compounds LCF and LCH (13). LCH was first reported following the concise synthesis of LCC as a by-product during synthesis. During the synthesis of LCC, its regioismer LCH was also synthesized using the acid-mediated Claisen-Schmidt condensation reaction as a key step (14).

Matrin 3 (Matr3) is a highly conserved nuclear matrix protein, which can bind to DNA and RNA through zinc finger domains (ZF1 and ZF2) and RNA recognition motifs (RRM1 and RRM2) (15). Matr3 is widely expressed in a variety of tissues and is known to be involved in DNA replication, apoptosis, transcription, translation, RNA processing, chromatin remodeling (16), and the stabilization of RNA and mRNA (17). The $\mathrm{Ca}^{2+} /$ calmodulin binding motif partially overlaps the RNA recognition motifs domain of Matr3, and Matr3 is also known to be degraded by various caspases, including caspase-3, $-5,-6,-7$ and -10 . The function of Matr3 can be regulated by calmodulin- and $\mathrm{Ca}^{2+}$-dependent interactions and caspase-mediated cleavage during apoptosis (18).

The present study investigated the anticancer effect of LCH on OSCCs, which induced cell apoptosis, cell cycle arrest and anchorage-independent cell transformation in OSCCs. The aim of the present study was to determine the efficacy of $\mathrm{LCH}$, which remains to be elucidated in oral cancer, and to suggest the potential for its development as a potential anticancer drug.

\section{Materials and methods}

Materials. Dulbecco's modified Eagle's medium (DMEM), fetal bovine serum (FBS), penicillin and streptomycin $(\mathrm{P} / \mathrm{S})$, and $0.05 \%$ trypsin-EDTA were purchased from Thermo Fisher Scientific, Inc. (Waltham, MA, USA). CNBr-activated Sepharose $^{\mathrm{TM}}$ 4B was purchased from GE Healthcare Life Sciences (Uppsala, Sweden). 4'-6-diamidino-2-phenylindole (DAPI) was purchased from Sigma-Aldrich; EMD Millipore (Billerica, MD, USA). The antibodies used included Matr3 (C-20; cat. no. sc-55723), cyclin D1 (M-20; cat. no. sc-718), p27 (C-19; cat. no. sc-528), B-cell lymphoma 2 (Bcl-2; C-2; cat. no. sc-7382), Bcl-2-extra large (Bcl-xL; H-5; cat. no. sc-8392), Bcl-2-associated X protein (Bax; N-20; cat. no. sc-493), and Bcl-2-associated death promotor (Bad; C-7; cat. no. sc-8044) from Santa Cruz Biotechnology, Inc. (Santa Cruz, CA, USA)' poly (ADP-ribose) polymerase (PARP; cat. no. 9542), survivin (71G4B7; cat. no. 2808) and caspase-3 (cat. no. 9662) from Cell Signaling Technology, Inc. (Danvers, MA, USA) and GAPDH (LF-PA0018) from Abcam
(Cambridge, UK). LCH was synthesized using the same method as that used in a previous study (14).

Cell culture. The HSC2 and HSC3 human OSCC cells were obtained from Hokkaido University (Hokkaido, Japan). The cells were cultured in DMEM containing 10\% heat-inactivated FBS, and $100 \mathrm{U} / \mathrm{ml}$ each $\mathrm{P} / \mathrm{S}$, and were incubated at $37^{\circ} \mathrm{C}$ with $5 \% \mathrm{CO}_{2}$ in humidified air.

3-(4,5-Dimethylthiazol-2-yl)-5-(3-carboxy-methoxyphenyl)2-(4-sulfophenyl)-2H-tetrazolium (MTS) assay. To investigate the effect of $\mathrm{LCH}$ on cell viability, the CellTiter $96^{\mathrm{TM}}$ Assay kit (Promega Corp., Madison, WI, USA) was used, according to the manufacturer's protocol. The HSC2 and HSC3 cells ( $3 \times 10^{3}$ cells/well) were cultured n 96 -well plates for $24 \mathrm{~h}$, and treated with $\mathrm{LCH}$ for 24 and $48 \mathrm{~h}$ at $37^{\circ} \mathrm{C}$. Subsequently, $20 \mu \mathrm{l}$ of MTS solution was added to each well, and the plates were reacted at $37^{\circ} \mathrm{C}$ for $4 \mathrm{~h}$. The optical density was measured at $490 \mathrm{~nm}$ using the Epoch $^{\mathrm{TM}}$ microplate spectrophotometer (BioTek Instruments, Inc., Winooski, VT, USA). Cell viability was calculated using the following equation: Optical density ratio of $\mathrm{LCH}$-treated sample/untreated sample x100 (\%).

DAPI staining. The HSC2 and HSC3 cells treated with $\mathrm{LCH}$ were collected, washed with cold $1 \mathrm{X}$ phosphate-buffered saline (PBS) and fixed with $100 \%$ methanol for $30 \mathrm{~min}$ at room temperature. Following fixation, the cells on the slide were stained with $2 \mu \mathrm{g} / \mathrm{ml}$ of DAPI solution. The stained cells were observed using a Nikon C2 Plus microscope (Nikon Corp., Tokyo, Japan).

Annexin V/propidium iodide (PI) staining. The HSC2 and HSC 3 cells were cultured and treated with $\mathrm{LCH}(0,10,20$ and $30 \mu \mathrm{M}$ ) for $48 \mathrm{~h}$. The adherent cells and floating cells were collected and washed with cold $1 \mathrm{X}$ PBS. The cells were then stained with the FITC Annexin V Apoptosis Detection kit (BD Biosciences, Franklin Lakes, NJ, USA) and analyzed using a fluorescence-activated cell sorter (FACS; BD Biosciences).

Cell cycle analysis. The HSC2 and HSC3 cells were cultured and treated with $\mathrm{LCH}$ at concentrations of 10,20 and $30 \mu \mathrm{M}$ for $48 \mathrm{~h}$. The cells were harvested, washed with cold 1X PBS, and fixed with $70 \%$ ethanol at $-20^{\circ} \mathrm{C}$ for $2 \mathrm{~h}$. The cells were then washed with 1X PBS and stained with RNase A and PI (BD Biosciences). They were then analyzed using a FACS, according to the manufacturer's protocol.

Western blot analysis. The HSC2 and HSC3 cells were harvested following treatment with $\mathrm{LCH}(10,20$ and $30 \mu \mathrm{M})$ for $48 \mathrm{~h}$. The proteins were then extracted using RIPA buffer (Thermo Fisher Scientific, Inc.) containing protease inhibitor cocktail (Roche Diagnostics, Basel, Switzerland). The concentration of the extracted protein was determined using a BCA Protein Assay kit (Thermo Fisher Scientific, Inc.) and the equivalent quantity of proteins were electrophoresed on 8, 10 and 15\% SDS-PAGE gels, and transferred onto a polyvinylidene fluoride (PVDF) membrane. The membranes were then reacted with the specific primary antibody $(1: 1,000$ to $1: 2,000)$ diluted in Tris-buffered saline (TBS) 
containing $0.1 \%$ Tween-20 (TBST) at $4^{\circ} \mathrm{C}$ overnight. The reacted membranes were washed three times for $10 \mathrm{~min}$ in TBST. Following incubation with a horseradish peroxidase (HRP)-labeled secondary antibody against anti-rabbit IgG (LF-SA8002; Ab Frontier, San Diego, CA, USA), anti-goat IgG (cat. no. sc-2020; Santa Cruz Biotechnology, Santa Cruz, CA, USA), anti-mouse IgG (cat. no. sc-2005; Santa Cruz Biotechnology) was diluted in TBST, the PVDF membrane was reacted with enhanced chemiluminescence reagent (Santa Cruz Biotechnology, Inc., Dallas, TX, USA) and then detected using ImageQuant LAS 4000 Mini system (GE Healthcare Life Sciences, Chalfont, UK) according to the manufacturer's protocol.

Pull-down assay. LCH was coupled to $\mathrm{CNBr}$-activated Sepharose $^{\mathrm{TM}}$ 4B matrix beads in $0.1 \mathrm{M} \mathrm{NaHCO}_{3}(\mathrm{pH} 8.3)$ containing $0.5 \mathrm{M} \mathrm{NaCl}$ overnight at $4{ }^{\circ} \mathrm{C}$, according to the manufacturer's protocol. The HSC2 and HSC 3 cell lysates were mixed with $\mathrm{LCH}$-conjugated Sepharose $4 \mathrm{~B}$ beads or with Sepharose 4B beads alone as a control. The binding of LCH and Matr3 was observed using western blot analysis.

Anchorage-independent cell transformation assay. The HSC2 and HSC 3 cells were treated with various concentrations of LCH in $1 \mathrm{ml}$ of $0.33 \%$ basal medium Eagle's agar over $3 \mathrm{ml}$ of $0.5 \%$ basal medium Eagle's agar containing $10 \%$ FBS. The cultures were maintained in a $37^{\circ} \mathrm{C}, 5 \% \mathrm{CO}_{2}$ incubator for 20 days. The colony numbers and sizes were measured under a microscope (Nikon C2 Plus system; Nikon Corp.). Respective experiments were performed in triplicate, and the average values were used.

Matr3 small interfering RNA (siRNA). siRNA sequences targeting Matr3 (matrin-3 siRNA; cat. no. sc-62604) and a non-targeting control (control siRNA; cat. no. sc-37007) were purchased from Santa Cruz Biotechnology. Transfection was performed according to the manufacturer's protocol. The HSC 2 and HSC 3 cells were cultured in 6-well plates and transfected with $50 \mathrm{nM}$ siRNA using siRNA transfection reagent (Lipofectamine 2000; Thermo Fisher Scientific,Inc.) according to the manufacturer's protocol. Following transformation for 24, 48 and $72 \mathrm{~h}$, the HSC2 and HSC 3 cells were used for western blot analysis, reverse transcription-polymerase chain reaction (RT-PCR) analysis, and the anchorage-independent cell transformation assay.

RT-PCR analysis. Total RNA was extracted from the cells using TRIzol ${ }^{\circledR}$ reagent (Thermo Fisher Scientific, Inc.). A total $5 \mu \mathrm{g}$ of RNA was used to synthesize cDNA using the

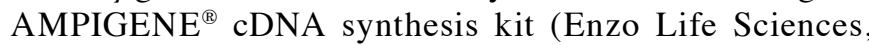
Farmingdale, NY, USA). cDNA was obtained by PCR amplication using $\beta$-actin-specific and Matr3-specific primers (as described below) for 30 cycles under the PCR conditions of $1 \mathrm{~min}$ at $95^{\circ} \mathrm{C}, 1 \mathrm{~min}$ at $58^{\circ} \mathrm{C}$ and $1 \mathrm{~min}$ at $72^{\circ} \mathrm{C}$. The $\beta$-actin primers used were as follows: Forward $5^{\prime}$-GTGGGGCGC CCCAGGCACCA-3' and reverse 5'-CTCCTTAATGTCACG CACGATTTC-3'. The Matr3 primers used were as follows: Forward 5'-TGGAGCAAGTCACAGTCGTC-3' and reverse 5'-TCTGCCTTTCTGCATGTGTC-3'. The PCR products were analyzed by $1 \%$ agarose gel electrophoresis.
Statistical analysis. The data are presented as the mean \pm standard deviation. Statistical analysis of the data was performed using the Prism 5.0 statistical package (GraphPad Software, Inc., La Jolla, CA, USA). The statistical significance of differences among groups were analyzed using one-way analysis of variance and Fisher's least significant difference post hoc test. $\mathrm{P}<0.05$ was considered to indicate a statistically significant difference. In the present study, the data are representative of three independent experiments performed in triplicate.

\section{Results}

Anticancer effect of LCH on OSCC cell lines. An MTS assay was performed to investigate the effect of $\mathrm{LCH}$ on the viability of OSCC cells (HSC2 and HSC 3 ). The HSC2 and HSC 3 cells were cultured in 96-well plates for $24 \mathrm{~h}$ and then treated with LCH $(0,10,20,30$ and $40 \mu \mathrm{M})$ for 24 and $48 \mathrm{~h}$, respectively. The results of MTS assay showed that the viability of the HSC2 and HSC 3 cells decreased, in a dose- and time-dependent manner, compared with that in the untreated group (Fig. 1A). The concentrations of 10,20 and $30 \mu \mathrm{M}$ of $\mathrm{LCH}$ were selected following confirmation of the significant viability inhibition of LCH on the HSC2 and HSC 3 cells. Following treatment with $\mathrm{LCH}$, the morphologies of the $\mathrm{HSC} 2$ and $\mathrm{HSC} 3$ were altered and the cell confluency decreased (Fig. 1B). The HSC2 and HSC 3 cells were treated with LCH, and DAPI staining was performed to determine the characteristics of apoptosis, including nuclear fragmentation and chromatin condensation. The percentage of apoptotic cells with respect to the total cell count is shown in Fig. 1C, showing an increase in the number of apoptotic cells in the cells treated with $\mathrm{LCH}$ compared with the DMSO-treated group. To investigate the changes of anchorage-independent colony formation by treatment with LCH in OSCC cells, a colony formation assay was performed. LCH markedly suppressed anchorage-independent colony formation in the HSC 2 and HSC 3 cells in a concentration-dependent manner (Fig. 1D and E).

LCH induces apoptosis in OSCC cell lines. The LCH-induced apoptosis and the Sub-G1 population of LCH-treated OSCC cells were increased compared with that in the untreated OSCC cells. Annexin V/PI double staining was performed to investigate whether LCH affects the induction of apoptosis in HSC2 and HSC3 cells. LCH induced the apoptosis of HSC2 and HSC 3 cells in a concentration-dependent manner. The percentages of total apoptotic HSC2 cells were 80.96, 86.92, and $89.52 \%$ following treatment with 10,20 and $30 \mu \mathrm{M} \mathrm{LCH}$, respectively, and the percentages of total apoptotic HSC 3 cells were $85.34,93.61$ and $95.38 \%$ following treatment with 10,20 and $30 \mu \mathrm{M}$, respectively (Fig. 2A). To investigate the effect of LCH on apoptosis, FACS analysis was performed by PI staining. In the on HSC2 cells, the percentage of the Sub-G1 population was $1.71 \%$ in the control cells, whereas treatment with 10,20 , and $30 \mu \mathrm{M}$ of LCH for $48 \mathrm{~h}$ led to values of 6.52 , 16.77 and $32.23 \%$. In the HSC3 cells, the percentage of the Sub-G1 population was $4.02 \%$ in the control, whereas treatment with LCH led to values of 11.39, 17.69 and 31.2\% (Fig. 2B).

Effect of LCH on Matr3 in OSCC cell lines. To determine whether LCH binds to Matr3, ex vivo pull-down 
A
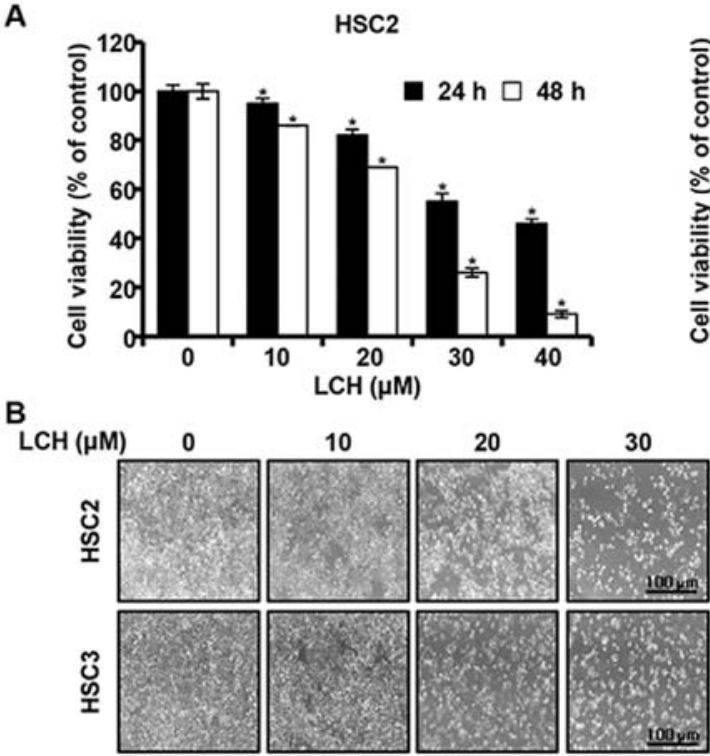

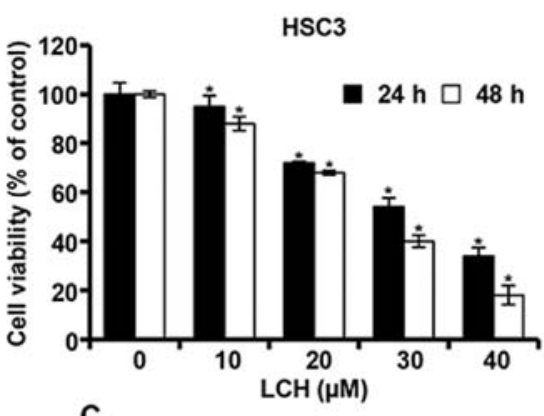

C

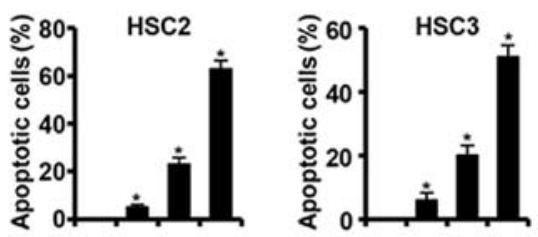

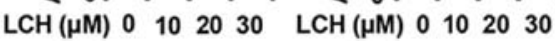

D

E
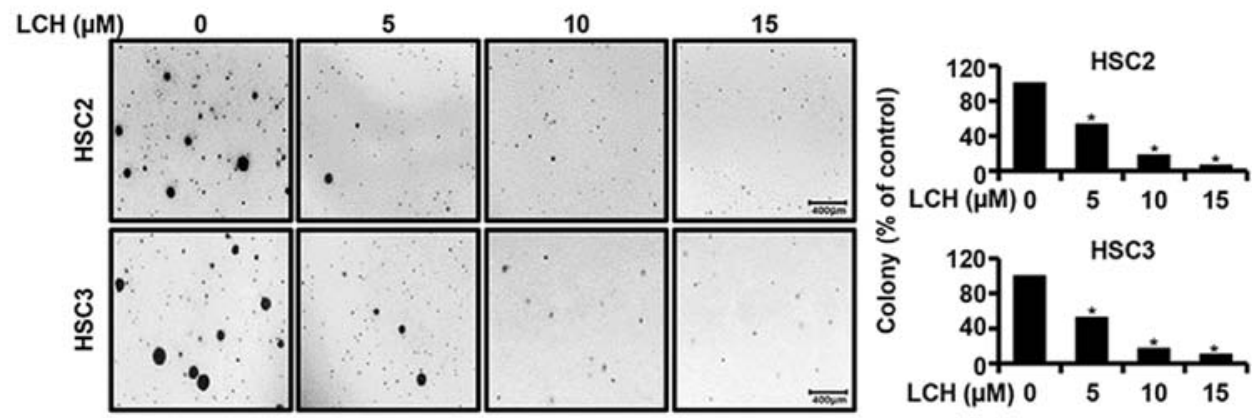

Figure 1. Anticancer effect of LCH on OSCC. (A) Viabilities of HSC2 and HSC3 OSCC lines treated with LCH (0-40 $\mu \mathrm{M})$ for 24 and $48 \mathrm{~h}$. Viability was measured using a 3-(4,5-dimethylthiazol-2-yl)-5-(3-carboxy-methoxyphenyl)-2-(4-sulfophenyl)-2H-tetrazolium assay kit. Data are presented as the mean \pm standard deviation. (B) Following treatment with LCH for $48 \mathrm{~h}$, morphological changes in OSCC cells were detected using an optical microscope. (C) Cell nuclei were stained with 4'-6-diamidino-2-phenylindole and images were captured with a fluorescence microscope. DNA fragmentation and nuclear condensation were quantified, and the data are presented as the mean \pm standard deviation $\left(n=3 ;{ }^{*} \mathrm{P}<0.05\right)$. (D) HSC2 and HSC 3 cells were treated with LCH $(5,10$ and $15 \mu \mathrm{M})$ in $1 \mathrm{ml}$ of $0.3 \%$ basal medium Eagle's agar containing $10 \%$ fetal bovine serum. All cells were incubated at $37^{\circ} \mathrm{C}$ in a $5 \% \mathrm{CO}_{2}$ incubator for 20 days and colonies were counted. (E) Numbers of colonies were calculated as the mean \pm standard deviation $\left(n=3\right.$; $\left.{ }^{*} \mathrm{P}<0.05\right)$. OSCC, oral squamous cell carcinoma; $\mathrm{LCH}$, licochalcone $\mathrm{H}$.

A
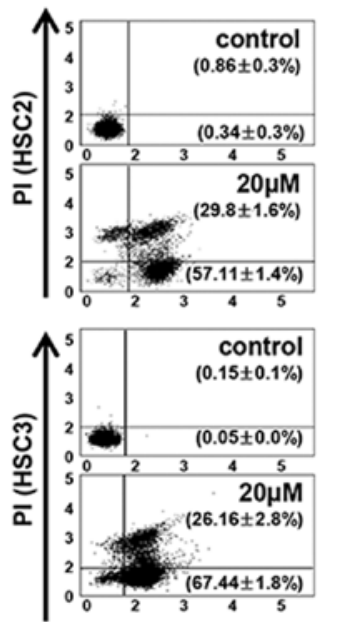

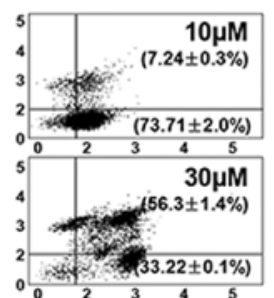

Annexin V
B
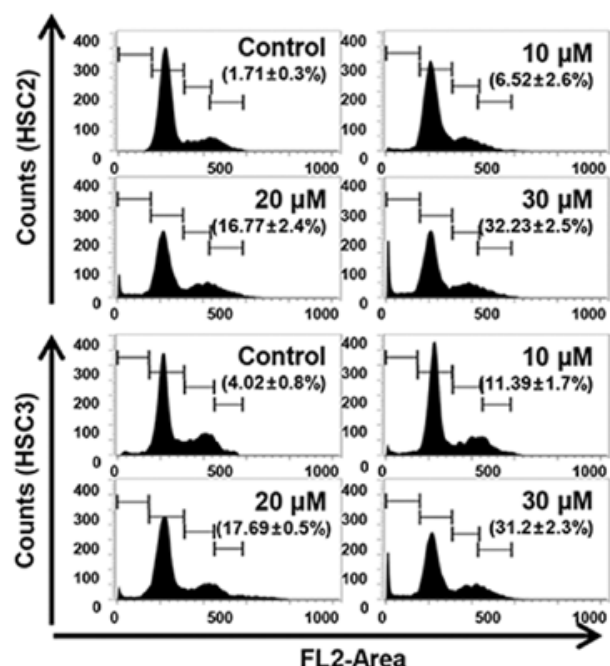

Figure 2. Effect of licochalcone $\mathrm{H}$ treatment on apoptosis in oral squamous cell carcinoma. (A) Cells with Annexin V/PI double staining were examined using a fluorescence-activated cell sorter. The graphs are representative of triplicate experiments, and the percentages of Annexin V-positive (lower-right quadrant) and Annexin V/PI double-positive (upper-right quadrant) are shown in the relevant quadrants. (B) Cell death analysis using PI staining and flow cytometry. The graph represents the proportion of Sub-G1 peak phase (apoptotic) cells. PI, propidium iodide. 
A

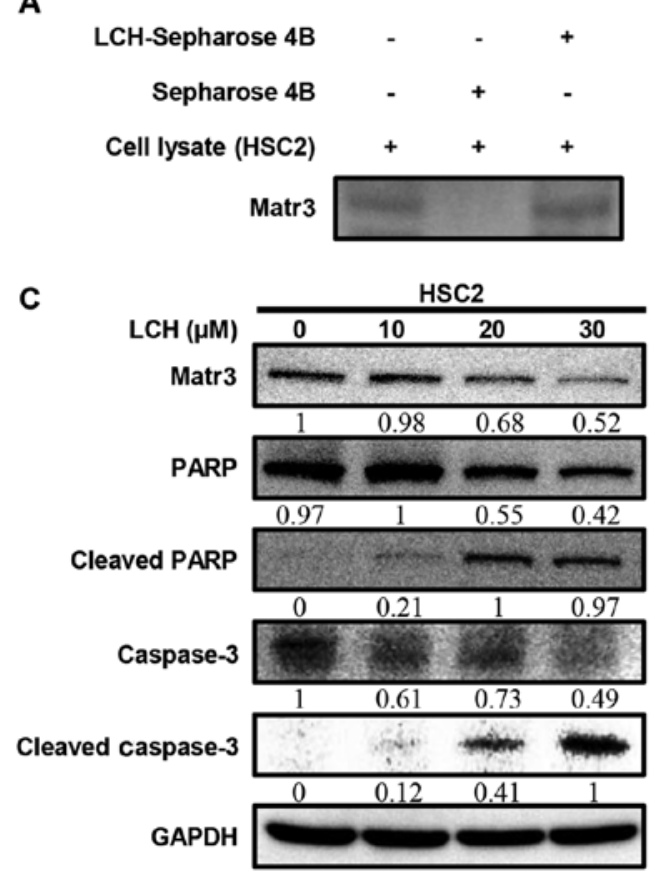

B
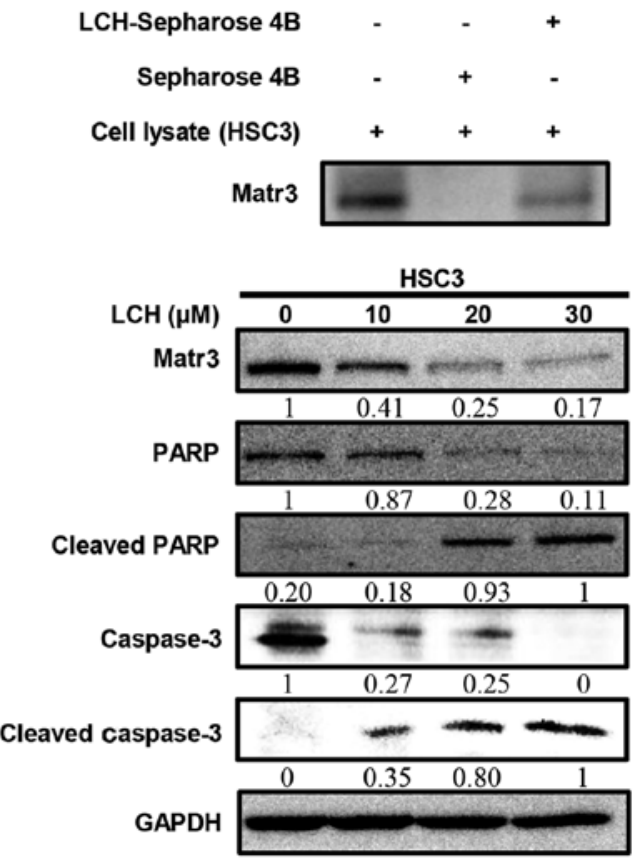

Figure 3. Effect of LCH on the expression of Matr3 proteins in the oral squamous cell carcinoma cell lines. Pull-down assays of LCH binding to Matr3 ex vivo. Whole (A) HCS2 and (B) HSC3 cell lysates were incubated with Sepharose 4B or LCH-Sepharose 4B beads overnight at $4^{\circ} \mathrm{C}$. Immunoblotting of pull-down products was performed with the anti-Matr3 antibody. HSC2 and HSC3 cells were seeded onto a cell culture plate for $24 \mathrm{~h}$ and treated with LCH (10,20 and $30 \mu \mathrm{M}$ ) for $48 \mathrm{~h}$. (C) Cells were harvested and western blot analysis was performed via SDS-PAGE. Expression of Matr3, PARP, cleaved PARP, caspase-3, cleaved caspase-3 and GAPDH were detected by western blot analysis using specific antibodies. GAPDH protein was used as the loading control. LCH, licochalcone H; Matr3, matrin 3; PARP, poly (ADP-ribose) polymerase.
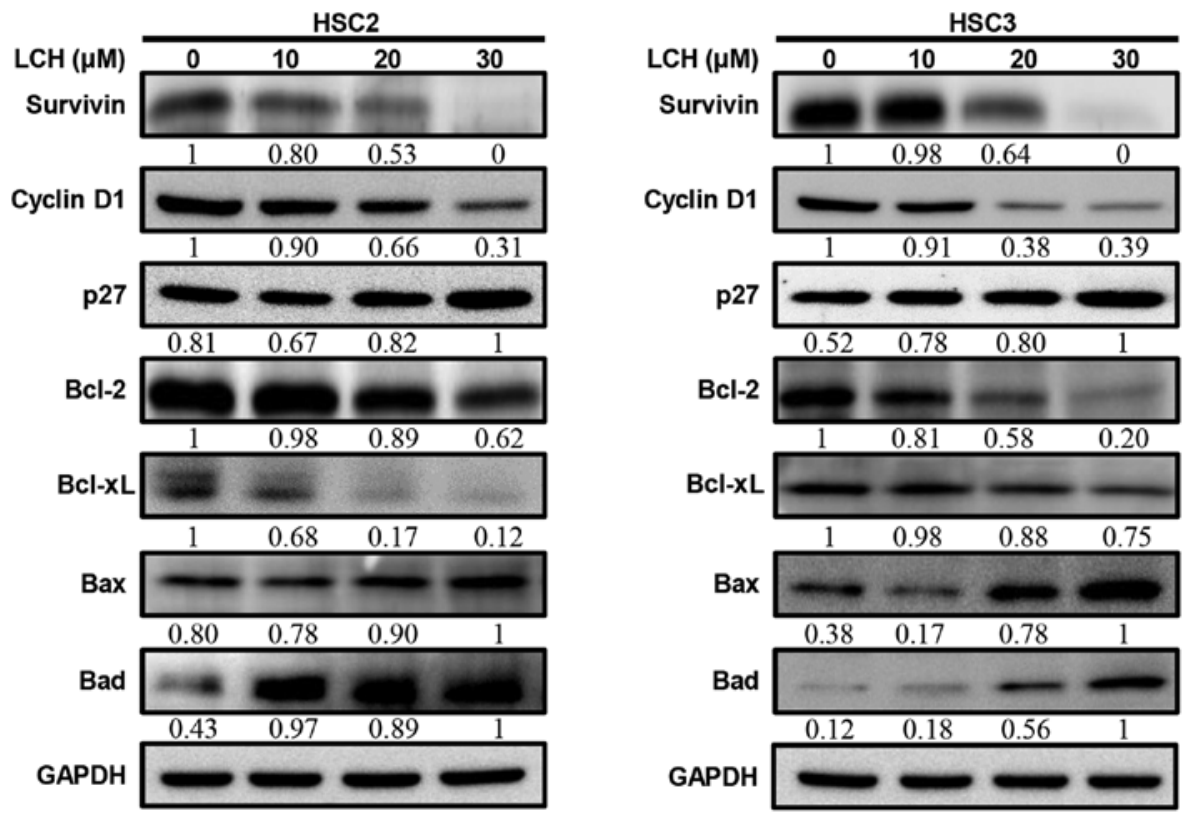

Figure 4. Effect of LCH on apoptosis regulatory proteins. HSC2 and HSC3 cells were seeded onto a cell culture plate for $24 \mathrm{~h}$, and treated with 10,20 and 30 $\mu \mathrm{M} \mathrm{LCH}$ for $48 \mathrm{~h}$. The proteins were then separated via SDS-PAGE and western blot analysis was performed using survivin, cyclin D1, p27, Bcl-2, Bcl-xL, Bax and Bad antibodies. GAPDH protein was used here as an internal control. LCH, licochalcone $\mathrm{H}$; Bcl-2, B-cell lymphoma 2; Bax, Bcl-2-associated X protein; Bad, Bcl-2-associated death promotor.

assays were performed. The HSC2 and HSC 3 cell lysates directly interacted with the LCH-Sepharose $4 \mathrm{~B}$ beads in vitro (Fig. $3 \mathrm{~A}$ and $\mathrm{B}$ ). The HSC2 and HSC3 cells were used to examine the effect of LCH on the expression of Matr3 and other apoptosis-related factors. The protein expression levels of the Matr3 in the HSC2 and HSC 3 cells treated with LCH were decreased in a dose-dependent manner (Fig. 3C). The cleavage of PARP and caspase-3, which are involved in the apoptotic pathway, increased in a dose-dependent manner (Fig. 3C). 
A

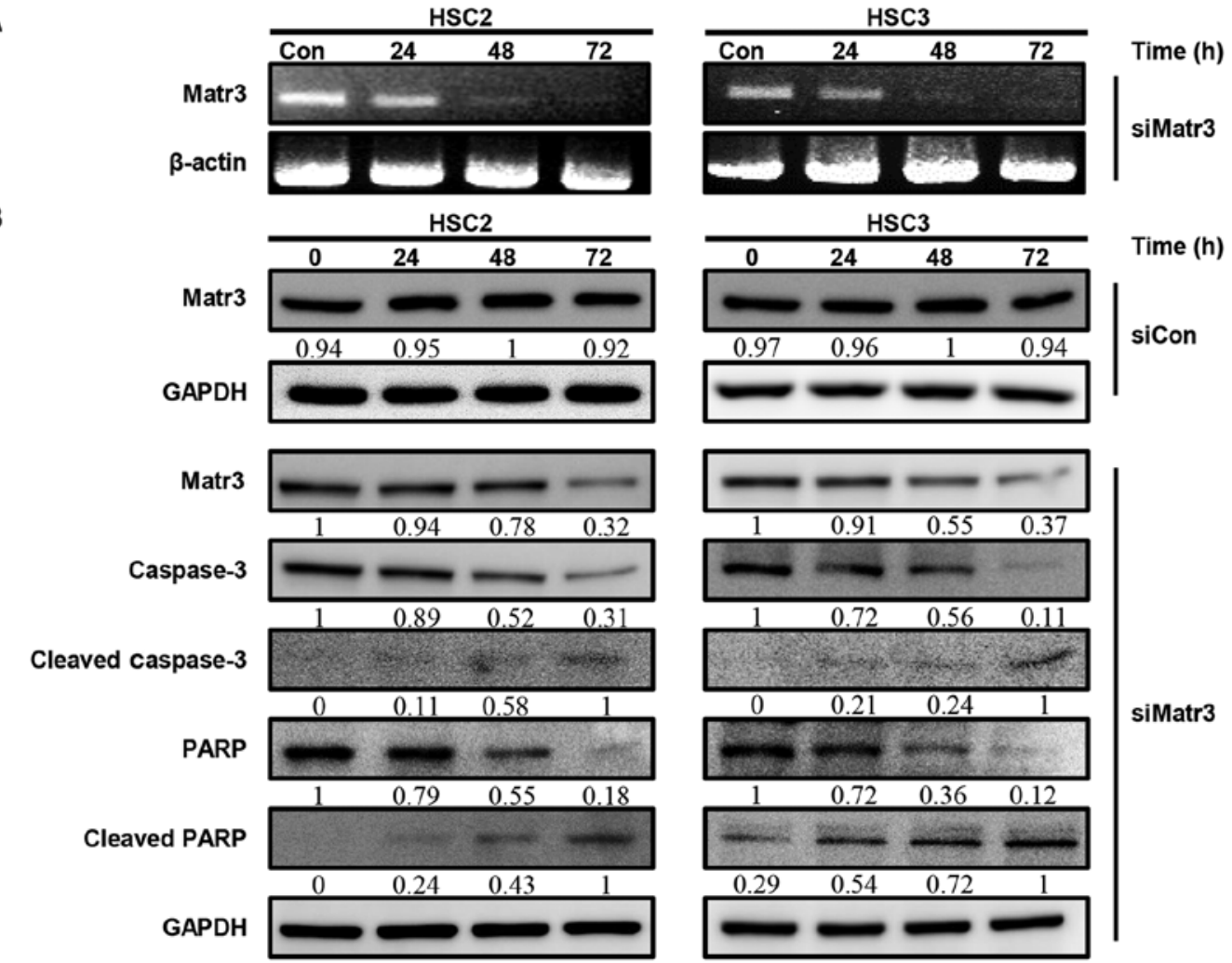

C
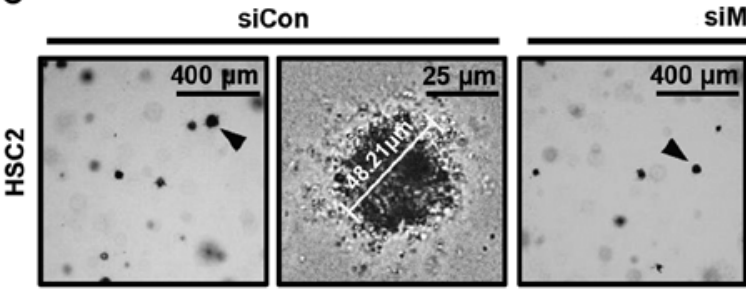

siMatr3
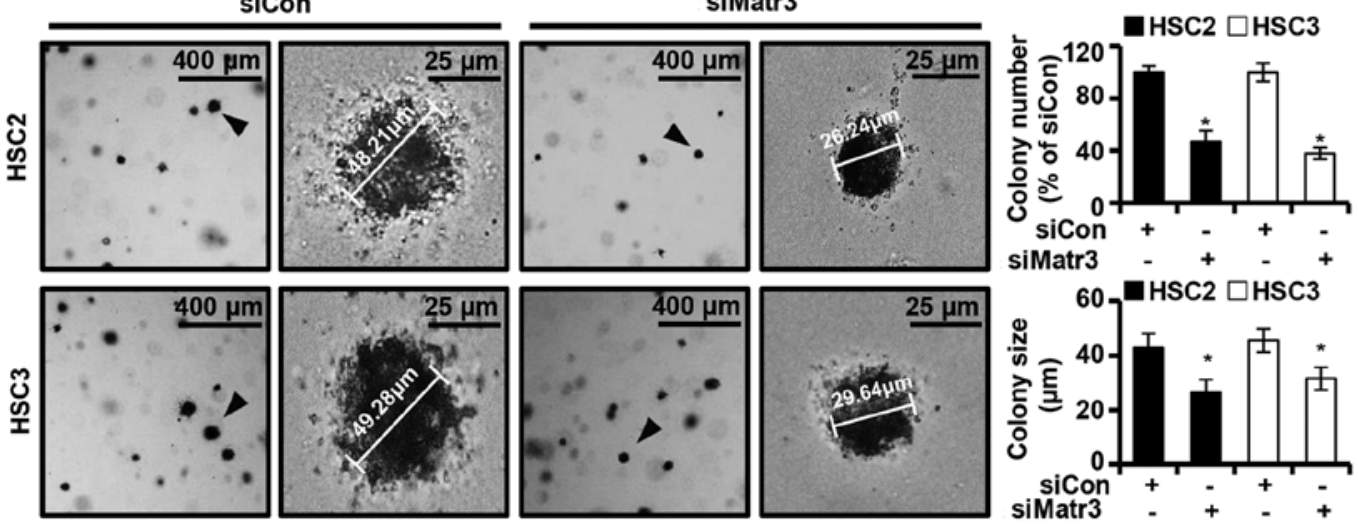

Figure 5. Knockdown of the expression of Matr3 results in induction of oral squamous cell carcinoma cell apoptosis. HSC2 and HSC3 cells were transfected with siCon or siMatr3. (A) mRNA expression level of Matr3 in each cell transfected with siCon or siMatr3 was analyzed by reverse transcription-polymerase chain reaction analysis. $\beta$-actin was used as a loading control. (B) siCon- or siMatr3-transfected HSC2 and HSC3 cell lysates were determined by western blot analysis using anti-Matr3, anti-caspase-3, anti-cleaved caspase-3, anti-PARP and anti-cleaved PARP antibodies. GAPDH was used as the loading control. (C) HSC2 and HSC3 cells were transfected with siCon or siMatr3 and soft agar assays were performed. Colony numbers and sizes were measured, and the results are expressed as the mean \pm standard deviation $\left({ }^{*} \mathrm{P}<0.05\right)$ for triplicate experiments. siMatr3, matrin 3-specific targeting siRNA; siCon, scrambled control siRNA; PARP, poly (ADP-ribose) polymerase.

LCH regulates apoptosis-related proteins in OSCC cell lines. The HSC2 and HSC3 cells were treated with $\mathrm{LCH}$ for $48 \mathrm{~h}$, and the Matr3 regulatory protein expression levels were analyzed by western blot analysis. Following treatment, the expression level of p27, which regulates the cell cycle, increased, and the expression levels of cyclin D1 and survivin decreased (Fig. 4A). Pro-survival proteins, Bcl-2 and Bcl-xL decreased and pro-apoptotic proteins Bax and Bad increased in a dose-dependent manner.

Role of Matr3 in OSCC cell lines through siRNA transfection. The present study investigated whether the expression of Matr3 affects the apoptosis of OSCC cells. A knocked-down version of Matr3 (siMatr3) was transiently transfected into HSC2 and HSC 3 cells, and the expression levels of the Matr3 gene and other apoptosis-related factors were examined at different post-transfection time-points $(24,48$ and $72 \mathrm{~h})$. It was found that siMatr3 regulated the mRNA and protein levels of Matr3, and the full length of caspase- 3 and PARP in a time-dependent manner (Fig. 5A and B). Also, no change was identified in Matr3 protein expression of HSC2 and HSC 3 in the control siMatr3 (siCon) group (Fig. 5B). Furthermore, siMatr3 significantly inhibited colony formation and reduced colony size in the HSC2 and HSC 3 cells (Fig. 5C). 


\section{Discussion}

Globally, cancer is the second leading cause of mortality in humans, and its incidence at younger ages is increasing (1). Although oral cancer has a lower incidence rate than other major types of cancer, including lung, breast and colorectal cancer, its treatment requires attention, as its incidence has gradually increased over the last 20 years worldwide (2). There have been substantial advances in treatment from drug development studies, specifically to hallmark signaling of cancer cell proliferation, growth, death, angiogenesis, invasion and metastasis (19). Previous studies have suggested that Matr3 is involved in DNA replication, RNA metabolism and nuclear retention of hyper-edited RNA, apoptosis, transcription and translation in HeLa cells, and is known to stabilize RNA and mRNA in 293T cells (15).It also has been reported that Matr3 regulates cell growth and proliferation by forming complexes with nucleoproteins that regulate pro- and anti-apoptotic signaling pathways (19). Matr3 is an RNA- and DNA-binding nuclear matrix protein found to be associated with degenerative diseases (21) and cancer (22).

The results of the present study showed that the chemotherapeutic agent LCH inhibited the carcinogenesis of oral cancer through the suppression of Matr3 protein. A previous study showed that the knockdown of Matr3 in endothelial cells induced predominantly necrosis but not apoptosis, indicating that Matr3 is involved in the control of cell proliferation and viability of endothelial cells, with Matr3 being a key regulator of endothelial cell survival (23). The results of the present study demonstrated that decreased Matr3 in OSCC cells induced apoptosis. Using a combination of siRNA techniques, it was found that Matr3 regulated apoptosis and colony formation in OSCC cells (Fig. 5). The expression levels of apoptotic factors PARP and caspase-3 were found to decrease, in contrast to the cleaved forms of PARP and caspase-3, which were increased in siMatr3 transfected cells (Fig. 5B). These results showed that Matr3 protein is important in the process of carcinogenesis in OSCC. The present study also established OSCC lines with downregulated Matr3 to identify the role of Matrin3 in OSCCs. However, it was not possible to establish Matr3-knockdown OSCC lines, as survival rate was low in Matr3-knockdown OSCC cells (data not shown).

A wide variety of LCs isolated from the roots of Glycyrrhiza inflate have shown various biological effects and/or chemopreventive potential (12). LCH has a 3,3-dimethylallyl group at C-5 in the $\mathrm{B}$ ring, unlike $\mathrm{LCC}$ at $\mathrm{C}-3$ in the $\mathrm{B}$ ring, and its structure is similar to that of LCA, with the exception of an allyl group. Studies have revealed that compounds with substituents at $\mathrm{C}-5$ in the $\mathrm{B}$ ring exhibit more beneficial biological effects $(24,25)$. To date, LCs have shown to exhibit various biological activities, and the anticancer effect of LCH is anticipated. The present study demonstrated that LCH inhibited the cell growth of HSC2 and HSC 3 human OSCC cells through the induction of apoptotic cell death and suppression of anchorage-independent colony formation via a decrease in the expression of Matr3. The half-maximal inhibitory concentration values were 36 and $23 \mu \mathrm{M}$ in HSC2 cells following treatment for 24 and $48 \mathrm{~h}$, respectively, and were 33 and $19 \mu \mathrm{M}$ in the HSC 3 cells following treatment for 24 and $48 \mathrm{~h}$, respectively. In order to clarify the association between LCH and Matr3, pull-down analysis was performed using
LCH-Sepharose-4B beads with OSCC cell lysates. As shown in Fig. 3A and B, LCH directly bound with Matr3 protein in the OSCC cells. $\mathrm{LCH}$ also significantly decreased the protein expression of Matr3 in HSC2 and HSC 3 cells (Fig. 3C). This result suggested that LCH directly targeted Matr3 in OSCC cells. LCH led to time-dependent and dose-dependent OSCC cell growth inhibition (Fig. 1A), which appeared to be due to its ability to induce the Sub-G1 population (Fig. 2B). The association between the cell cycle and apoptosis provides evidence that manipulation of the cell cycle may either prevent or induce an apoptotic response (25). LCH inhibited cyclin D1 and increased p27 in a dose dependent manner (Fig. 4). During the G1 to S progression of the cell cycle, cyclin D1 and cyclin-dependent kinase inhibitor p27 $7^{\mathrm{kip}}$ are involved in growth arrest resulting from DNA damage, cell senescence, and terminal differentiation or cell cycle entry, progression, and apoptosis (27). The present study analyzed LCH-mediated apoptosis using Annexin V/PI staining. When apoptosis is induced, phosphatidyl serine, which exists inside the cell membrane, is externally exposed and Annexin V binds to the released phosphatidyl serine. Early-apoptosis is positive for Annexin V staining as PI does not penetrate the cell membrane; however, as apoptosis progresses, the integrity of the plasma membrane is impaired and PI can pass through the membrane for staining (28). The present study confirmed that early-apoptosis and late-apoptosis were increased following treatment with LCH (Fig. 2A). LCH exhibited an apoptotic effect on the HSC2 and HSC3 cells. Anti-apoptotic proteins, including Bcl-2 and Bcl-xL, can directly or indirectly suppress apoptosis, and apoptosis is induced by the overexpression of Bax and Bad (29). The present study examined the protein expression of Bcl-xL, Bcl-2, Bax, and Bad in HSC2 and HSC3 cells (Fig. 4), LCH significantly downregulated the protein expression of $\mathrm{Bcl}-2$ and $\mathrm{Bcl}-\mathrm{xL}$ and upregulated the protein expression of Bax and Bad, compared with expression levels in the control.

Taken together, these results suggested that $\mathrm{LCH}$ regulated Matr3, and ultimately caused apoptosis in OSCC. Therefore, LCH offers potential to be developed as a promising therapeutic agent for OSCC. Additionally, Matr3 was essential for OSCC proliferation, and the downregulation of Matr3 induced apoptosis, suggesting that Matr3 may be an effective therapeutic target for oral cancer.

\section{Acknowledgements}

Not applicable.

\section{Funding}

The present study was supported by a grant (grant no. 16182MFDS391) from the Korean Ministry of Food and Drug Safety in 2017 and the Cooperative Research Program for Agriculture Science and Technology Development (project no. PJ012704012018)' of the National Institute of Animal Science, Rural Development Administration, Republic of Korea. This study was also carried out with the support of the Cooperative Research Program for Agriculture Science and Technology Development (project no. PJ013842)', Rural Development Administration, Republic of Korea. 


\section{Availability of data and materials}

The datasets used during the present study are available from the corresponding author upon reasonable request.

\section{Authors' contributions}

JHSh and JIC conceived the project and designed all experiments. SHN, GY and JIC designed and performed the cell experiments, and JHSe, HNO, SSC, HK and HWC performed and analyzed the biological experiments. JIC, JHSh, SHN and GY wrote the manuscript. All authors read and approved the manuscript and agree to be accountable for all aspects of the research in ensuring that the accuracy or integrity of any part of the work are appropriately investigated and resolved.

\section{Ethics approval and consent to participate}

Not applicable.

\section{Patient consent for publication}

Not applicable.

\section{Competing interests}

The authors declare that they have no competing interests.

\section{References}

1. Jemal A, Bray F, Center MM, Ferlay J, Ward E and Forman D: Global cancer statistics. CA Cancer J Clin 61: 69-90, 2011.

2. Warnakulasuriya S: Global epidemiology of oral and oropharyngeal cancer. Oral Oncol 45: 309-316, 2009.

3. Bagan J, Sarrion G and Jimenez Y: Oral cancer: Clinical features. Oral Oncol 46: 414-417, 2010.

4. Schantz SP and Yu GP: Head and neck cancer incidence trends in young Americans, 1973-1997, with a special analysis for tongue cancer. Arch Otolaryngol Head Neck Surg 128: 268-274, 2002.

5. Feller LL, Khammissa RR, Kramer BB and Lemmer JJ: Oral squamous cell carcinoma in relation to field precancerisation: Pathobiology. Cancer Cell Int 13: 31, 2013.

6. Jung KW, Won YJ, Oh CM, Kong HJ, Cho H, Lee JK, Lee DH and Lee KH: Prediction of cancer incidence and mortality in Korea, 2016. Cancer Res Treat 48: 451-457, 2016.

7. Cho JH, Lee RH, Jeon YJ, Shin JC, Park SM, Choi NJ, Seo KS, Yoon G, Cho SS, Kim KH, et al: Role of transcription factor Sp1 in the 4-O-methylhonokiol-mediated apoptotic effect on oral squamous cancer cells and xenograft. Int J Biochem Cell Biol 64: 287-297, 2015

8. Harvey AL: Natural products in drug discovery. Drug Discov Today 13: 894-901, 2008.

9. Watkins R, Wu L, Zhang C, Davis RM and Xu B: Natural product-based nanomedicine: Recent advances and issues. Int J Nanomedicine 10: 6055-6074, 2015.
10. Cragg GM and Newman DJ: Plants as a source of anti-cancer agents. J Ethnopharmacol 100: 72-79, 2005.

11. Newman DJ and Cragg GM: Natural products as sources of new drugs over the 30 years from 1981 to 2010. J Nat Prod 75: 311-335, 2012.

12. Wang ZY and Nixon DW: Licorice and cancer. Nutr Cancer 39: $1-11,2001$.

13. Yoon G, Jung YD and Cheon SH: Cytotoxic allyl retrochalcone from the roots of Glycyrrhiza inflata. Chem Pharm Bull (Tokyo) 53: 694-695, 2005.

14. Wang Z, Cao Y, Paudel S, Yoon G and Cheon SH: Concise synthesis of licochalcone $\mathrm{C}$ and its regioisomer, licochalcone $\mathrm{H}$. Arch Pharm Res 36: 1432-1436, 2013.

15. Nakamura A, Osonoi T and Terauchi Y: Relationship between urinary sodium excretion and pioglitazone-induced edema. J Diabetes Investig 1: 208-211, 2010.

16. Zeitz MJ, Malyavantham KS, Seifert B and Berezney R: Matrin 3: Chromosomal distribution and protein interactions. J Cell Biochem 108: 125-133, 2009.

17. Salton M, Elkon R, Borodina T, Davydov A, Yaspo ML, Halperin E and Shiloh Y: Matrin 3 binds and stabilizes mRNA. PLoS One 6: e23882, 2011.

18. Valencia CA, Ju W and Liu R: Matrin 3 is a $\mathrm{Ca}^{2+} /$ calmodulin-binding protein cleaved by caspases. Biochem Biophys Res Commun 361: 281-286, 2007.

19. Hanahan D and Weinberg RA: Hallmarks of cancer: The next generation. Cell 144: 646-674, 2011.

20. Przygodzka P, Boncela J and Cierniewski CS: Matrin 3 as a key regulator of endothelial cell survival. Exp Cell Res 317: 802-811, 2011.

21. Coelho MB, Attig J, Bellora N, König J, Hallegger M, Kayikci M, Eyras E, Ule J and Smith CW: Nuclear matrix protein Matrin3 regulates alternative splicing and forms overlapping regulatory networks with PTB. EMBO J 34: 653-668, 2015.

22. Chaudhary R, Gryder B, Woods WS, Subramanian M, Jones MF, Li XL, Jenkins LM, Shabalina SA, Mo M, Dasso M, et al: Prosurvival long noncoding RNA PINCR regulates a subset of p53 targets in human colorectal cancer cells by binding to Matrin 3. Elife 6: pii: e23244, 2017.

23. Patrycja P, Joanna B and Czeslaw SC: Matrin 3 as a key regulator of endothelial cell survival. Exp Cell Res 317: 802-811, 2011.

24. Yoon G, Lee W, Kim SN and Cheon SH: Inhibitory effect of chalcones and their derivatives from Glycyrrhiza inflata on protein tyrosine phosphatase 1B. Bioorg Med Chem Lett 19: 5155-5157, 2009.

25. Liu Z, Lee W, Kim SN, Yoon G and Cheon SH: Design, synthesis, and evaluation of bromo-retrochalcone derivatives as protein tyrosine phosphatase 1B inhibitors. Bioorg Med Chem Lett 21: 3755-3758, 2011.

26. Schafer KA: The cell cycle: A review. Vet Pathol 35: 461-478, 1998.

27. Ilyin GP, Glaise D, Gilot D, Baffet G and Guguen-Guillouzo C: Regulation and role of p21 and p27 cyclin-dependent kinase inhibitors during hepatocyte differentiation and growth. Am J Physiol Gastrointest Liver Physiol 285: G115-G127, 2003.

28. Oh H, Yoon G, Shin JC, Park SM, Cho SS, Cho JH, Lee MH, Liu K, Cho YS, Chae JI, et al: Licochalcone B induces apoptosis of human oral squamous cell carcinoma through the extrinsicand intrinsic-signaling pathways. Int J Oncol 48: 1749-1757, 2016.

29. Zhai D, Jin C, Huang Z, Satterthwait AC and Reed JC: Differential regulation of Bax and Bak by anti-apoptotic Bcl-2 family proteins Bcl-B and Mcl-1. J Biol Chem 283: 9580-9586, 2008. 